\title{
Comparison of severity predictive rules for hospitalised nursing home-acquired pneumonia in Korea: a retrospective observational study
}

\author{
Jong-Chan Lee', Hee-Jin Hwang ${ }^{2,3}$, Yo-Han Park', Jun-Hyeon Joe ${ }^{4}$, Jae-Ho Chung', \\ *Sang-Hwan Kim ${ }^{2,3}$
}

\author{
' Department of Internal Medicine, Kwangdong University College of Medicine, Goyang, Korea \\ ${ }^{2}$ Geriatric Center \& Department of Family Medicine, Kwangdong University College of Medicine, Goyang, Korea \\ ${ }^{3}$ Geriatric Center, Myongji Hospital, Goyang, Korea \\ ${ }^{4}$ Department of Internal Medicine, Incheon Sarang Hospital, Incheon, Korea
}

Received 21st July 2012; revised 10th November 2012; accepted 27th November 2012; online 15th March 2013

\begin{abstract}
Background: Nursing home-acquired pneumonia (NHAP) is the leading cause of death among long-term care residents.

Aims: To compare current scoring indices (NHAP model score, Pneumonia Severity Index (PSI), CURB-65 (confusion, urea nitrogen, respiratory rate, blood pressure, age $>65$ years) and SOAR (systolic blood pressure, oxygenation, age, respiratory rate)) in predicting mortality and admission to the intensive care unit (ICU) in patients with NHAP.

Methods: This retrospective observational study was conducted between July 2008 and June 2011 using data from the Korean Nursing Home Networks. Two hundred and eight nursing home residents were hospitalised with pneumonia in one general hospital. The primary outcome measure was 30-day all-cause mortality. Secondary outcome measures were intensive respiratory or vasopressor support (IRVS), and severe pneumonia (ICU admission or IRVS).

Results: PSI class $V$ showed the highest Youden index (0.45), specificity (66.7\%), positive predictive value (PPV, 40.0\%), negative predictive value (NPV, 91.5\%), and area under the curve (AUC, 0.73) for 30-day mortality. For severe pneumonia, PSI class $V$ showed the highest Youden index (0.40), specificity (72.8\%), PPV (62.2\%), NPV (77.1\%), and AUC (0.70). Similarly, PSI class V showed the highest Youden index (0.35), specificity (68.3\%), PPV (51.1\%), NPV (80.5\%), and AUC (0.69) for IRVS.

Conclusions: The PSI has superior discriminatory power in predicting all three clinical outcomes (30-day mortality, severe pneumonia, and IVRS) compared with the NHAP model score, CURB-65 and SOAR.

(C) 2013 Primary Care Respiratory Society UK. All rights reserved.

J-C Lee et al. Prim Care Respir J 2013; 22(2): 149-154

http://dx.doi.org/10.4104/pcrj.2013.00011
\end{abstract}

Keywords aged, nursing homes, pneumonia

\section{See linked editorial by Netuveli on pg 139}

\section{Introduction}

Nursing home-acquired pneumonia (NHAP) is the leading cause of death among long-term care residents and the second most common cause of transfer to acute care facilities where it accounts for $2-18 \%$ of patients hospitalised for pneumonia. ' Nursing home residents develop pneumonia approximately 10 times more frequently than older adults in the community. ${ }^{2}$ Their hospitalisation rate is reported to be nearly 30 times higher than those in the community, ${ }^{3}$ with an estimated 30-day mortality rate of $10-56 \%$ depending on severity and initial response to therapy. ${ }^{4.5}$

Few models have been developed specifically to measure the severity of NHAP. Naughton et al. derived a model (NHAP model score) to predict 30-day all-cause mortality in nursing home residents that included four predictors (respiratory rate $>30 / \mathrm{min}$, pulse $>125 / \mathrm{min}$, altered mental status and history of dementia). ${ }^{6}$ van der Steen et al. developed a severity model in Dutch residents of nursing homes with dementia and pneumonia that was validated in a US population. The assumption is that the van der Steen model

\footnotetext{
* Corresponding author:Sang-Hwan Kim, Geriatric Center \& Department of Family Medicine, Kwandong University College of Medicine Hospital, 407-1 Jakjeon 2-dong, Gyeyang-gu, Incheon metropolitan city, Republic of Korea. Tel: +82-32-551-0114 Fax: +82-32-545-0975 E-mail: fanink@naver.com
} 
applies to all nursing home residents and not just those with dementia, but this has not been determined.?

Several severity indices for community-acquired pneumonia (CAP) can be used to decide the site of care and guide both general management and antibiotic treatment by relating a number of clinical and laboratory features to significant outcomes such as mortality. Comparative studies of indices for CAP in NHAP have recently been carried out. ${ }^{8,9}$ Indices included in these studies were the Pneumonia Severity Index (PSI), ${ }^{10}$ CURB-65 criteria (confusion, urea nitrogen, respiratory rate, blood pressure, age $>65$ years $)^{11}$ and the SOAR criteria (systolic blood pressure, oxygenation, age, respiratory rate). ${ }^{12}$ Each scoring system has its strengths and drawbacks, and the nursing home-focused index (NHAP model score) was not included in the abovementioned studies. We therefore sought to compare the performance of the indices (NHAP model score, PSI, CURB-65, and SOAR) in predicting mortality, intensive care unit (ICU) admission and intensive respiratory or vasopressor support (IRVS) in patients with NHAP.

\section{Methods}

\section{Study population}

This study was conducted between July 2008 and June 2011 using data from the Korean Nursing Home Networks. This network was established in July 2008 for the purpose of constructing a healthcare system for nursing home residents and to provide consumer-centred welfare and medical services. Currently, the network is composed of 35 nursing homes with one general hospital in Incheon metropolitan city and 30 nursing homes with one university hospital in Gyeonggi province. The present study was part of the LOVE (Long-term care of Old people Via KorEan nursing home network) study, which was initiated to gauge the value of the network in the early detection, management, and prevention of geriatric diseases among nursing home residents. All patients aged $>65$ years who had been admitted to one general hospital and who fulfilled the definition of NHAP were included in the study. This retrospective observational study was approved by the institutional review board of Myongji Hospital to review and publish information from the patients' records (Myongji Hospital IRB number 10-073) and the board dispensed with the need for permission.

\section{Data collection}

Data collected at admission included age, gender, admission from a nursing home, coexisting illness, symptoms, and clinical parameters. The clinical parameters included blood pressure, pulse rate, respiratory rate, percutaneous oxygen saturation, tympanic temperature, and mental confusion. A patient's mental status was clinically assessed by the attending emergency physician and mental confusion was defined as a Glasgow Coma Scale score of $<15$ or a new onset of disorientation to time, place or person for practical purposes. Additional data collected for all patients immediately after admission included laboratory results (complete blood count, arterial blood gas, glucose, electrolytes and urea), radiographic findings reported by the radiologists, and outcome variables (requirement for ICU admission or mechanical ventilation, length of stay in hospital, and all-cause 30-day mortality). The primary outcome measure was 30-day all-cause mortality. Secondary outcome measures were IRVS (i.e. invasive or non-invasive mechanical ventilation or infusions of vasopressors for blood pressure support) and severe pneumonia (ICU admission or IRVS).

\section{Definition of pneumonia and severity scores}

A clinical diagnosis of pneumonia required the presence of new radiographic infiltrates and at least one of the following clinical signs

Table 1. Baseline characteristics of patients with NHAP

\begin{tabular}{|c|c|}
\hline Characteristic & Mean \pm SD or $\mathrm{N}(\%)$ \\
\hline Age (years) & $80.0 \pm 9.0$ \\
\hline Sex (men) & $118(56.7)$ \\
\hline \multicolumn{2}{|l|}{ Co-morbidities } \\
\hline Cerebrovascular disease & $74(35.6)$ \\
\hline Heart failure & $13(6.3)$ \\
\hline Hypertension & $129(62.0)$ \\
\hline Diabetes mellitus & $47(22.6)$ \\
\hline Dementia & $109(52.4)$ \\
\hline Parkinson disease & $19(9.1)$ \\
\hline Chronic lung disease & $24(11.5)$ \\
\hline Cancer & $11(5.3)$ \\
\hline Previous fracture & $39(18.8)$ \\
\hline \multicolumn{2}{|l|}{ Physical examination findings } \\
\hline Confusion & $129(62.0)$ \\
\hline Systolic BP <90mmHg & $34(16.3)$ \\
\hline Diastolic BP $\leq 60 \mathrm{mmHg}$ & $75(36.1)$ \\
\hline Pulse rate $\geq 120 / \mathrm{min}$ & $19(9.1)$ \\
\hline Respiratory rate $\geq 30 / \mathrm{min}$ & $15(7.2)$ \\
\hline Body temperature $<35^{\circ} \mathrm{C}$ or $\geq 40^{\circ} \mathrm{C}$ & $3(1.4)$ \\
\hline O2 saturation $\leq 90 \%$ & $73(35.1)$ \\
\hline \multicolumn{2}{|l|}{ Laboratory and radiographic findings } \\
\hline WBC count $<4,000$ cells $/ \mathrm{mm}^{3}$ & $8(3.8)$ \\
\hline Haematocrit $<30 \%$ & $46(22.1)$ \\
\hline Platelet count $<105$ cells $/ \mathrm{mm}^{3}$ & $4(1.9)$ \\
\hline Glucose level $\geq 250 \mathrm{mg} / \mathrm{dL}$ & $15(7.2)$ \\
\hline BUN level >19mg/dL & $134(64.4)$ \\
\hline BUN level >30mg/dL & $70(33.7)$ \\
\hline Sodium level $<30 \mathrm{mmol} / \mathrm{L}$ & $24(11.5)$ \\
\hline Arterial $\mathrm{pH}<7.35$ & $23(11.1)$ \\
\hline $\mathrm{PaO}_{2}<60 \mathrm{mmHg}$ & $90(43.3)$ \\
\hline $\mathrm{SaO}_{2}<90 \%$ & $61(29.3)$ \\
\hline $\mathrm{PaO}_{2} / \mathrm{FiO}_{2}<250$ & $86(41.3)$ \\
\hline Multilobar involvement & $51(24.5)$ \\
\hline Pleural effusion & $19(9.1)$ \\
\hline \multicolumn{2}{|l|}{ Clinical outcomes } \\
\hline ICU admission & $55(26.4)$ \\
\hline Received ventilation & $34(16.3)$ \\
\hline Received vasopressor support & $63(30.3)$ \\
\hline 30-day mortality & $46(22.1)$ \\
\hline Hospital length of stay (days) & $14.5 \pm 14.0$ \\
\hline
\end{tabular}

$\mathrm{BP}=$ blood pressure, $\mathrm{BUN}=$ blood urea nitrogen, $\mathrm{FiO}_{2}=$ fraction of inspired oxygen, $\mathrm{ICU}=$ intensive care unit, NHAP=nursing home-acquired pneumonia,

$\mathrm{PaO}_{2}=$ arterial oxygen pressure, $\mathrm{SaO}_{2}=$ arterial oxygen saturation, $\mathrm{SD}=$ standard deviation, $\mathrm{WBC}=$ white blood cell. 
or symptoms to be included in this study: cough, pleuritic chest pain, fever $\geq 38^{\circ} \mathrm{C}\left(100.5^{\circ} \mathrm{F}\right)$, purulent sputum, respiratory rate $\geq 25 / \mathrm{min}$, or altered breath sounds on auscultation (rales, rhonchi, or dullness to percussion). ${ }^{13}$

Over the study period, all consecutive patients admitted to the hospital through their nursing home with pneumonia were eligible. Patients with hospital-acquired pneumonia that developed after being hospitalised for more than 48 hrs or within 14 days of leaving the hospital were not included. Additionally, immunocompromised patients such as those with neutropenia after chemotherapy or human immunodeficiency virus (HIV) infection and those who had undergone transplantations were excluded. Of 272 consecutive patients admitted with a provisional diagnosis of NHAP, 208 (76\%) were included in the study; 64 patients (14\%) were excluded either because they had exclusion criteria or a non-NHAP diagnosis.

Severity of pneumonia was evaluated using the prediction rule calculated according to the NHAP model score, ${ }^{6}$ PSI, ${ }^{10}$ CURB-65 criteria, ${ }^{11}$ and SOAR criteria. $^{12}$

\section{Statistical analyses}

A statistical software package (SPSS for Windows Version 15.0; SPSS, Chicago, IL, USA) was used for all statistical comparisons. Descriptive statistics of demographic and clinical variables were presented by frequencies, percentage or mean \pm standard deviation (SD). $\chi^{2}$ tests were used to compare categorical variables. The Youden index (sensitivity + specificity - 1), sensitivity, specificity, positive predictive value (PPV) and negative predictive value (NPV) of each rule were compared. The overall accuracies in predicting $30-$ day mortality, receiving IRVS and severe pneumonia were calculated as the area under the receiver operating characteristic curve (AUC); $p$ values $<0.05$ were regarded as statistically significant.

\section{Results}

\section{Baseline characteristics}

The baseline characteristics of the 208 patients included in the study are shown in Table 1; 118 (56.7\%) were male and the mean age was $80.0 \pm 9.0$ years. Forty-six patients $(22.1 \%)$ died within 30 days of admission, 55 (26.4\%) needed ICU admission and 34 (16.3\%)

Table 2. Relationship between the severity of NHAP and four predictive tools

\begin{tabular}{|c|c|c|c|c|}
\hline Risk groups & $\begin{array}{l}\text { No. of patients } \\
(\mathrm{N}=208)\end{array}$ & $\begin{array}{l}\text { 30-day mortality } \\
(\mathrm{N}=46)\end{array}$ & $\begin{array}{l}\text { Severe pneumonia } \\
(\mathrm{N}=83)\end{array}$ & $\begin{array}{l}\text { IRVS } \\
(\mathrm{N}=69)\end{array}$ \\
\hline \multicolumn{5}{|l|}{ PSI } \\
\hline III & $21(10.1)$ & $1(4.8)$ & $6(28.6)$ & $2(9.5)$ \\
\hline IV & $97(46.6)$ & $9(9.3)$ & $21(21.6)$ & $21(21.6)$ \\
\hline V & $90(43.3)$ & $36(40.0)$ & $56(62.2)$ & $46(51.1)$ \\
\hline $\mathrm{p}$ Value & & $<0.001$ & $<0.001$ & $<0.001$ \\
\hline 2 & $68(32.7)$ & $6(8.8)$ & $15(22.1)$ & $16(23.5)$ \\
\hline 3 & $74(35.6)$ & $21(28.4)$ & $35(47.3)$ & $28(37.8)$ \\
\hline 4 & $38(18.3)$ & $14(36.8)$ & $20(52.6)$ & $19(50.0)$ \\
\hline 5 & $6(28.8)$ & $3(50.0)$ & $6(100.0)$ & $4(66.7)$ \\
\hline $\mathrm{p}$ Value & & 0.001 & $<0.001$ & 0.002 \\
\hline 3 & $26(12.5)$ & $11(42.3)$ & $19(73.1)$ & $19(73.1)$ \\
\hline 4 & $2(1.0)$ & $1(50.0)$ & $2(100.0)$ & $0(0.0)$ \\
\hline$p$ Value & & 0.013 & $<0.001$ & $<0.001$ \\
\hline \multicolumn{5}{|c|}{ NHAP model score } \\
\hline 0 & $43(20.7)$ & $2(4.7)$ & $7(16.3)$ & $8(18.6)$ \\
\hline 1 & $80(38.5)$ & $20(25.0)$ & $33(41.3)$ & $28(35.0)$ \\
\hline 2 & $63(30.3)$ & $15(23.8)$ & $24(38.1)$ & $20(31.7)$ \\
\hline 3 & $11(5.3)$ & $4(36.4)$ & $9(81.8)$ & $6(54.5)$ \\
\hline 4 & $7(3.4)$ & $5(71.4)$ & $7(100.0)$ & $4(57.1)$ \\
\hline 5 & $4(1.9)$ & $0(0.0)$ & $3(75.0)$ & $3(75.0)$ \\
\hline
\end{tabular}

Values are $\mathrm{N}(\%)$.

CURB-65 criteria=confusion, urea nitrogen, respiratory rate, blood pressure, age $>65$ years; IRVS=intensive respiratory or vasopressor support; NHAP=nursing home-acquired pneumonia; PSI=Pneumonia Severity Index; severe pneumonia=admission to intensive care unit or IRVS; SOAR criteria=systolic blood pressure, oxygenation, age, respiratory rate. 
received mechanical ventilation. The mean length of stay in hospital was $14.5 \pm 14.0$ days. Hypertension was the most common chronic disease $(62.0 \%)$ in study subjects, followed by dementia (52.4\%), cerebrovascular disease (35.6\%), diabetes mellitus (22.6\%), previous fracture $(18.8 \%)$, chronic lung disease $(11.5 \%)$, Parkinsons disease (9.1\%), heart failure (6.3\%) and cancer (5.3\%).

\section{Physical, laboratory and radiological findings}

The three most common physical findings were confusion (62.0\%), decreased $(\leq 60 \mathrm{mmHg})$ diastolic blood pressure $(36.1 \%)$ and decreased ( $\leq 90 \%)$ peripheral oxygen saturation (35.1\%). The three most common laboratory findings were blood urea nitrogen level $>19 \mathrm{mg} / \mathrm{dL}$ (64.4\%), arterial oxygen pressure $\left(\mathrm{PaO}_{2}\right)<60 \mathrm{mmHg}$ (43.3\%), and $\mathrm{PaO}_{2} / \mathrm{FiO}_{2}<250$ (41.3\%). Multilobar involvement was found in 51 patients (24.5\%) on chest x-ray and pleural effusion was found in 19 (9.1\%) - see Table 1.

\section{Comparisons of clinical outcomes}

Table 2 shows patient distribution and the corresponding clinical outcomes (30-day mortality, severe pneumonia, and need for IRVS) for each risk score of the four predictive rules. All four predictive rules showed an increasing risk of death, severe pneumonia and IRVS with increasing numbers of risk factors, which was statistically significant. The PSI and CURB-65 classified a significantly larger proportion of patients (43.3\% and $28.8 \%$, respectively) as the highest score group compared with SOAR (1.0\%) and the NHAP model score (1.9\%). The 30 -day mortality rate of the highest score group was $40.0 \%$ in PSI, $50.0 \%$ in CURB-65, 50.0\% in SOAR, and $0 \%$ in the NHAP model score, but the rate of the second highest group of the NHAP model score was $71.4 \%$. All patients in the highest CURB-65 and SOAR score groups developed severe pneumonia (ICU admission or IRVS).

\section{Comparison of predictive accuracy}

The calculated sensitivity and specificity and corresponding AUCs of each rule in identifying 30-day mortality, severe pneumonia, and IRVS are shown in Table 3. PSI class $V$ showed the highest Youden index (0.45), specificity (66.7\%), PPV (40.0\%), NPV (91.5\%), and AUC (0.73) for 30-day mortality. For severe pneumonia, PSI class $\vee$ showed the highest Youden index (0.40), specificity (72.8\%), PPV (62.2\%), NPV (77.1\%), and AUC (0.70). Similarly, PSI class $V$ showed the highest Youden index (0.35), specificity (68.3\%), PPV (51.1\%), NPV (80.5\%), and AUC (0.69) for IRVS. The PSI had a superior discriminatory power in predicting all three clinical outcomes (30-day mortality, severe pneumonia and IVRS) compared with CURB-65, SOAR, and the NHAP model score.

\section{Discussion}

\section{Main findings}

We evaluated 208 nursing home residents hospitalised with

Table 3. Comparison of the accuracy of assessment tools in predicting 30-day mortality, severe pneumonia, and IRVS of patients with NHAP

\begin{tabular}{|c|c|c|c|c|}
\hline & PSI class V & CURB- $65 \geq 3$ & SOAR $\geq 2$ & NHAP model score $\geq 2$ \\
\hline \multicolumn{5}{|l|}{ 30-day mortality } \\
\hline Youden index & 0.45 & 0.33 & 0.22 & 0.15 \\
\hline Sensitivity (\%) & 78.3 (65.9 to 90.6) & $82.6(71.2$ to 94.0$)$ & $67.4(53.3$ to 81.5$)$ & $52.2(37.2$ to 67.2$)$ \\
\hline Specificity (\%) & 66.7 (59.3 to 74.0$)$ & 50.6 (42.8 to 58.4$)$ & 54.3 (46.6 to 62.1$)$ & 62.3 (54.8 to 69.9$)$ \\
\hline PPV (\%) & 40.0 (29.7 to 50.3$)$ & $32.2(23.6$ to 40.8$)$ & 29.5 (20.7 to 38.4$)$ & $28.2(18.5$ to 38.0$)$ \\
\hline NPV (\%) & 91.5 (86.4 to 96.6$)$ & $91.1(85.1$ to 97.1$)$ & 85.4 (78.5 to 92.4$)$ & 82.1 (75.2 to 89.0$)$ \\
\hline $\mathrm{AUC}$ & $0.73(0.65$ to 0.81$)$ & $0.69(0.61$ to 0.77$)$ & $0.64(0.54$ to 0.73$)$ & $0.64(0.55$ to 0.72$)$ \\
\hline \multicolumn{5}{|c|}{ Severe pneumonia } \\
\hline Youden index & 0.40 & 0.28 & 0.22 & 0.18 \\
\hline Sensitivity (\%) & 67.5 (57.2 to 77.8$)$ & 73.5 (63.8 to 83.2) & 63.9 (53.3 to 74.4$)$ & $51.8(40.8$ to 62.8$)$ \\
\hline Specificity (\%) & 72.8 (64.9 to 80.7$)$ & $54.4(45.5$ to 63.3$)$ & 58.4 (49.6 to 67.2$)$ & $66.4(58.0$ to 74.8$)$ \\
\hline PPV (\%) & 62.2 (52.0 to 72.4$)$ & $51.7(42.5$ to 60.8$)$ & 50.5 (40.8 to 60.2$)$ & 50.6 (39.7 to 61.4) \\
\hline NPV (\%) & 77.1 (69.4 to 84.8$)$ & $75.6(66.5$ to 84.6$)$ & 70.9 (62.0 to 79.8$)$ & 67.5 (59.1 to 75.9$)$ \\
\hline $\mathrm{AUC}$ & $0.70(0.62$ to 0.77$)$ & $0.66(0.58$ to 0.73$)$ & 0.65 (0.57 to 0.73$)$ & $0.67(0.59$ to 0.74$)$ \\
\hline \multicolumn{5}{|l|}{ IRVS } \\
\hline Youden index & 0.35 & 0.26 & 0.24 & 0.10 \\
\hline Sensitivity (\%) & 66.7 (55.3 to 78.1$)$ & 73.9 (63.3 to 84.5$)$ & 66.7 (55.3 to 78.1$)$ & 47.8 (35.7 to 59.9) \\
\hline Specificity (\%) & $68.3(60.5$ to 76.2$)$ & $51.8(43.4$ to 60.2$)$ & $57.6(49.2$ to 65.9$)$ & $62.6(54.4$ to 70.7$)$ \\
\hline PPV (\%) & 51.1 (40.6 to 61.6$)$ & 43.2 (34.2 to 52.3) & 43.8 (34.2 to 53.5$)$ & $38.8(28.2$ to 49.4$)$ \\
\hline NPV (\%) & 80.5 (73.3 to 87.8$)$ & $80.0(71.6$ to 88.4$)$ & 77.7 (69.5 to 85.8$)$ & 70.7 (62.6 to 78.9$)$ \\
\hline$A \cup C$ & $0.69(0.61$ to 0.76$)$ & $0.67(0.59$ to 0.74$)$ & $0.66(0.58$ to 0.74$)$ & $0.60(0.52$ to 0.68$)$ \\
\hline
\end{tabular}

Values in brackets are $95 \%$ confidence intervals.

$A U C=$ area under the curve (ROC curve analysis); CURB-65 criteria=confusion, urea nitrogen, respiratory rate, blood pressure, age $>65$ years; IRVS=intensive respiratory or vasopressor support; NHAP=nursing home-acquired pneumonia; NPV=negative predictive value; $\mathrm{PPV}=$ positive predictive value; $P S I=$ pneumonia severity index; severe pneumonia=admission to intensive care unit or IRVS; SOAR criteria=systolic blood pressure, oxygenation, age, respiratory rate; Youden index=sensitivity + specificity -1 . 
pneumonia at one general hospital without entry barrier of tertiary care facilities and compared the relationships between the severities of NHAP scored by three prediction tools (PSI, CURB-65, and SOAR) with the NHAP model score. There was a useful predictability of severity of NHAP in PSI class. We defined the severe groups in each category as PSI class V, CURB-65 $\geq 3$, SOAR $\geq 2$, and NHAP model score $\geq 2$. Concerning 30-day mortality, PSI class $V$ showed the highest Youden index (0.45), specificity (66.7\%), PPV (40.0\%), NPV (91.5\%), and AUC (0.73). PSI class $\vee$ showed a similar tendency for the highest predictabilities for severe pneumonia and IRVS.

\section{Strengths and limitations of this study}

Several limitations must be considered in the interpretation of our results. The first limitation is the retrospective design. Secondly, our study was conducted on a relatively less number of patients versus other large clinical studies but sufficient to produce clinical outcomes. Third, there is a difference in the referral system between Korea and other countries. In a study of NHAP in the USA, of 280 patients treated initially in the nursing home, 175 (62.5\%) were treated with an oral agent whereas 105 (37.5\%) were treated initially with a parenteral (intramuscular or intravenous) antibiotic. ${ }^{6}$ In our study population pneumonia was mostly treated in hospital because parenteral antibiotics and imaging studies including chest x-ray are not available in Korean nursing homes.

\section{Interpretation of findings in relation to previously published work}

NHAP affects the most fragile and debilitated residents in long-term care settings and is associated with considerable morbidity and mortality. ${ }^{14}$ Prediction rules may be useful adjuncts for clinical decision-making. Hutt and Kramer $^{15}$ recommend hospitalisation if two or more of the following are present: oxygen saturation $<90 \%$ on room air, systolic blood pressure $<90 \mathrm{mmHg}$, respiratory rate $>30$ breaths/min, necessity for $3 \mathrm{~L} / \mathrm{min}$ of oxygen, unstable chronic lung disease, heart failure, diabetes, patient unable to be aroused if previously conscious, and new or increased agitation.

A number of existing prediction rules aim to stratify CAP into different risk categories based on combinations of different prognostic variables. The application of these rules is focused mainly on CAP. Few studies have compared the prognostic ability of CAP prediction rules for severe NHAP. A study of NHAP compared the sensitivity, specificity, PPV, and NPV of predicting severe pneumonia by five different predictive rules (PSI, CURB-65, M-ATS, R-ATS, España rule) and evaluated the usefulness of PSI and CURB-65 in NHAP. ${ }^{8}$ The investigators concluded that the PSI and CURB-65 are useful tools for identifying less severe NHAP. It was conducted at a university teaching hospital emergency department and admissions of pneumonia via outpatient departments were not enrolled in the study. El-Solh et al. ${ }^{9}$ evaluated 457 nursing home residents hospitalised with pneumonia at two university-affiliated tertiary care facilities and found that the need for ICU care was better identified with the SOAR model than with the other scoring rules (CURB, CURB-65, and CRB-65) in patients with NHAP.

Naughton et al. ${ }^{6}$ derived a model to predict 30-day mortality in patients with NHAP which demonstrated increasing mortality with increasing score. These results were consistent with those of our study. However, the CAP models tested (PSI, CURB-65, and SOAR) were marginally better than the NHAP model score in predicting the three outcomes evaluated. The model of Naughton et al. was derived from a cohort where three-quarters of patients with NHAP were not hospitalised, but most of the patients with pneumonia in our cohort were initially treated in hospital. In addition, the Naughton model was derived from pneumonia in the winter season (from November to April) but we investigated pneumonia occurring in all four seasons.

Implications for future research, policy and practice The PSI had a superior discriminatory power in predicting all three clinical outcomes (30-day mortality, severe pneumonia, and IVRS) of hospitalised patients with NHAP in Korea compared with the NHAP model score, CURB-65, and SOAR. The PSI contains several variables which need in-hospital evaluation such as arterial $\mathrm{pH}, \mathrm{PO}_{2}<60$, serum sodium level, haematocrit, serum glucose level, blood urea nitrogen level, and pleural effusion on the chest x-ray. The difficulty lies in checking those variables in general nursing homes that lack a laboratory and imaging tools. It is therefore necessary to develop an easier and more accurate prediction tool for severe pneumonia that is applicable in the setting of nursing homes.

\section{Conclusions}

The PSI has better discriminatory power in predicting all three clinical outcomes (30-day mortality, severe pneumonia, IVRS) than the NHAP model score, CURB-65, and SOAR.

\section{Handling editor Gopal Netuveli Statistical review Gopal Netuveli}

Conflicts of interest The authors declare that they have no conflicts of interest in relation to this article.

Contributorship $\mathrm{J}-\mathrm{CL}$ and $\mathrm{H}-\mathrm{JH}$ contributed equally to this work as first authors. J-CL, Y-HP, J-HJ and J-HC contributed to acquisition of subjects/data and the preparation of manuscript. $\mathrm{H}-\mathrm{JH}$ contributed to the study concept and design and preparation of the manuscript. S-HK contributed to the study concept and design, analysis and interpretation of the data and preparation of the manuscript. Funding This research was supported by the Basic Science Research Program through the National Research Foundation of Korea (NRF) funded by the Ministry of Education, Science and Technology (2011-0012207).

\section{References}

1. Ronald LA, McGregor MJ, McGrail KM, et al. Hospitalization rates of nursing home residents and community-dwelling seniors in British Columbia. Can J Aging 2008;27:109-15. http://dx.doi.org/10.3138/cja.27.1.109

2. Muder RR. Approach to the problem of pneumonia in long-term care facilities Compr Ther 2000;26:255-62. http://dx.doi.org/10.1007/s12019-000-0027-4

3. Marrie TJ. Pneumonia in the long-term-care facility. Infect Control Hosp Epidemiol 2002;23:159-64. http://dx.doi.org/10.1086/344281

4. Thompson RS, Hall NK, Szpiech M, Reisenberg LA. Treatments and outcomes of nursing-home-acquired pneumonia. J Am Board Fam Pract 1997;10:82-7.

5. Mehr DR, Zweig SC, Kruse RL, et al. Mortality from lower respiratory infection in nursing home residents. A pilot prospective community-based study. J Fam Pract 1998;47:298-304.

6. Naughton BJ, Mylotte JM, Tayara A. Outcome of nursing home-acquired pneumonia: derivation and application of a practical model to predict 30 day mortality. J Am Geriatr Soc 2000;48:1292-9.

7. van der Steen JT, Mehr DR, Kruse RL, et al. Predictors of mortality for lower respiratory infections in nursing home residents with dementia were validated transnationally. J Clin Epidemio/ 2006;59:970-9

http://dx.doi.org/10.1016/j.jclinepi.2005.12.005

8. Man SY, Graham CA, Chan SS, et al. Disease severity prediction for nursing home 
acquired pneumonia in the emergency department. Emerg Med J 2011;28:104650. http://dx.doi.org/10.1136/emj.2010.107235

9. El-Solh AA, Alhajhusain A, Abou Jaoude P, Drinka P. Validity of severity scores in hospitalized patients with nursing home acquired pneumonia. Chest 2010;138:1371-6. http://dx.doi.org/10.1378/chest.10-0494

10. Fine MJ, Auble TE, Yealy DM, et al. A prediction rule to identify low-risk patients with community-acquired pneumonia. $N$ Engl J Med 1997;336:243-50 http://dx.doi.org/10.1056/NEJM199701233360402

11. Lim WS, van der Eerden MM, Laing $R$, et al. Defining community acquired pneumonia severity on presentation to hospital: an international derivation and validation study. Thorax 2003;58:377-82.

http://dx.doi.org/10.1136/thorax.58.5.377
12. Myint PK, Kamath AV, Vowler SL, Maisey DN, Harrison BDW. Severity assessment criteria recommended by the British Thoracic Society (BTS) for community-acquired pneumonia (CAP) and older patients. Should SOAR (systolic blood pressure, oxygenation, age and respiratory rate) criteria be used in older people? A compilation study of two prospective cohorts. Age Ageing 2006;35:286-91. http://dx.doi.org/10.1093/ageing/afj081

13. Jackson MM, Fierer J, Barrett-Connor $\mathrm{E}$, et al. Intensive surveillance for infections in a three-year study of nursing home patients. Am J Epidemiol 1992;135:685-96.

14. Mylotte JM. Nursing home acquired pneumonia. Clin Infect Dis 2002;35:1205-11. http://dx.doi.org/10.1086/344281

15. Hutt E, Kramer AM. Evidence-based guidelines for management of nursing home acquired pneumonia. J Fam Pract 2002;51:709-16.

\section{Available online at http://www.thepcrj.org}

\section{$7^{\text {th }}$ World Conference of the International Primary Care Respiratory Group (IPCRG)}

\section{A Breath of Fresh Air: Multiple Morbidities and Integration 21-24 May 2014}

\section{Hilton Hotel, Athens}

- Internationally-renowned primary care speakers

- Symposia on primary care case-finding, diagnosis and management

- Cutting-edge real life primary care research from the leading respiratory units around the world

- Practical workshops to update your skills for everyday practice

- Opportunities to network with like-minded

colleagues from around the world - we expect at least 45 countries

Contact e-mail address ipcrg2014@mci-group.com

\section{www.jpcrg2014.org}

The IPCRG is a charity registered in Scotland, working internationally. It is in collaborative relations with WONCA and is the WONCA Europe respiratory Special Interest Group.

The 2014 conference is supported by ELEGEIA, the Greek Association of General Practitioners.
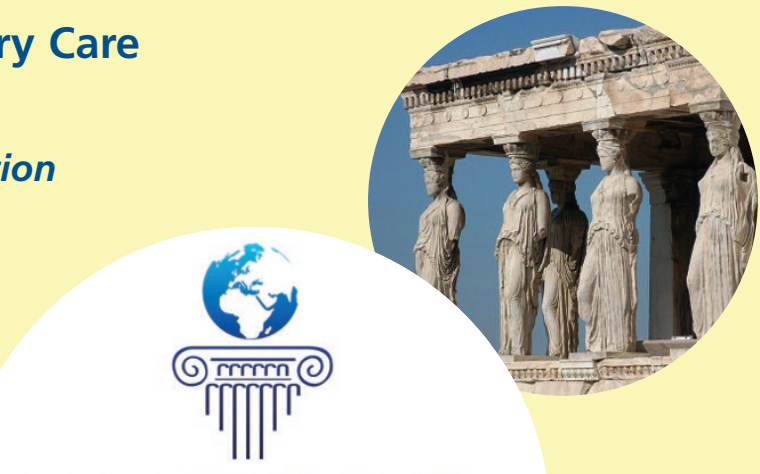

\section{7thIPCRG}

WORLD CONFERENCE

$21 \mathrm{ST}-24 \mathrm{TH} M A Y 2014$

Athens
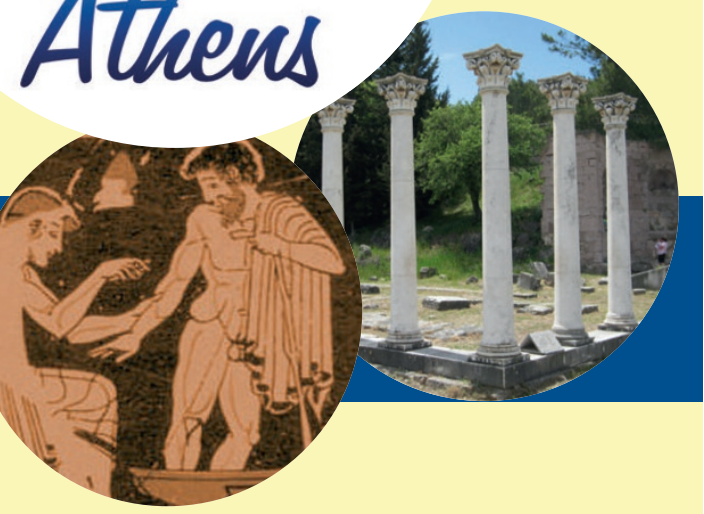